\title{
Preparation and Characterization of a High Strength Self-repairing Galactomannan Hydrogel
}

\author{
Jiufang Duan,* Yuxue Gao, Yirong Huang, Lin Li, and Jianxin Jiang
}

\begin{abstract}
Autonomous self-repairing properties can prolong the service life of materials. In this paper, galactomannan hydrogel with high mechanical strength was prepared by graft copolymerization of galactomannan with acrylamide and octadecyl methacrylate in aqueous solution. The microstructure, water absorption property, self-healing behavior, and mechanical properties of the hydrogels were investigated using Fourier transform infrared spectroscopy (FTIR) and scanning electron microscope (SEM), etc. The galactomannan hydrogel had the highest tensile strength of $49 \mathrm{KPa}$ and strain of $3000 \%$. The water absorption reached $2340 \%$, and the removal rate of methylene blue was more than $80 \%$. Galactomannan hydrogels demonstrated significant self-healing properties. The cut hydrogel was quite effective in self-repairing in a few minutes, and the selfrepairing strength increased with increasing contact time of hydrogel cut surfaces. The healing efficiency of fracture strain could reach $92.7 \%$ of the original sample in $10 \mathrm{~h}$. The maximum water absorption of hydrogel reached $2340 \%$. The maximum removal rate of methylene blue by hydrogel reached $80.5 \%$, and the maximum adsorption capacity was 19.3 $\mathrm{mg} / \mathrm{g}$. The novelty of the work lies in octadecyl methacrylate being used for galactomannan cross-linking with the ability to self-repair after fracture. The galactomannan self-healing hydrogel has potential in water treatment and sealing technology.
\end{abstract}

Keywords: Galactomannan; Cross-linking; Self-healing; Mechanical strength; Hydrophobic aggregation

Contact information: MOE Engineering Research Center of Forestry Biomass Materials and Bioenergy, Beijing Forestry University, Beijing 100083, China; *Corresponding author: duanjiu99@163.com

\section{INTRODUCTION}

Galactomannan is an abundant biopolymer derived from renewable resources locust bean gum, carob gum, tara gum, and guar gum. These naturally occurring renewable galactomannans are polymers with a $\beta$-(1-4)-D-mannose backbone and D-galactose side units linked at $\alpha-(1-6)$ (Chaires-Martinez et al. 2008). The species and extraction method can affect the mannose/galactose ratio in galactomannan. Galactomannan shows properties of emulsibility, film-forming, and low concentration aqueous solution with high viscosity. Galactomannan and its derivatives have been widely used as thickeners, stabilizers, foaming agents, and flocculants in the food, drug, textile, oil, paper, and cosmetics industries (Mukherjee et al. 2018; Paixao et al. 2018; Saurabh et al. 2018; Wang et al. 2018). Common water treatment technologies include adsorption (Wang et al. 2017), photocatalysis (Zhang et al. 2017, 2018), microwave catalysis (Wang et al. 2018), etc. There are many commonly used adsorbents for water treatment, such as activated carbon, double hydroxides, metal-organic framework and adsorption resin (Yang et al. 2018; Zhang et al. 2019). Galactomannan hydrogel is an important adsorption resin. The traditional modification methods for galactomannan include enzymatic hydrolysis, graft 
polymerization, cross-linking, and functional group derivation, such as oxidation, carboxymethylation, esterification, and etherification. The galactomannan solution cannot form a gel by itself. However, it synergizes with other polymers such as agarose, alginate, xanthan gum, casein, and starch (Ali and Husain 2018; Bak and Yoo 2018) to form a "complex" by increasing the viscosity of the solution. The aqueous solution of galactomannan and its derivatives form a gel with boron, titanium, zirconium, and chemical crosslinking (Li et al. 2018).

A high mechanical strength hydrogel with self-healing ability can greatly improve the service life and reliability of carrier materials such as cartilage and tissue engineering scaffolds. Usually, high mechanical strength of a hydrogel can be achieved by covalent cross-linking. At present, glutaraldehyde, N,N-methylene bisacrylamide, dichlorodimethylsilane, epichlorohydrin, and borax are commonly used as crosslinking agents in galactomannan modification (Chaurasia et al. 2006; Shi et al. 2011; Tang et al. 2013). This method of enhancing mechanical properties of hydrogel can lead to very limited movement of molecular chains. The self-healing properties of hydrogels are achieved by reversible interactions between polymer molecular chains and functional groups on the polymer molecular chains (Duan et al. 2014a,b; Taylor and In Het Panhuis 2016). The reversible interactions of polymer molecular chains include hydrophobically associated hydrogels, dipole-dipole enhanced hydrogels, ionically cross-linked hydrogels, hydrogen bonds, and dynamic covalent bonding hydrogels (Deng et al. 2012; Tuncaboylu et al. 2012; Cui et al. 2013; Wei et al. 2013; Bai et al. 2014; Chirila et al. 2014; Barcan et al. 2015; Bizien et al. 2015; Wei et al. 2015). Cross-linking is an effective method for reducing the viscosity and swelling power of galactomannan by increasing the molecular weight and the formation of cross-linkages (Cunha et al. 2005; Bahamdan et al. 2007). Therefore, studying the role of hydrophobic force in improving the mechanical strength and self-repairing property of the melon gel material is an important question (Duan et al. 2017).

To date, making a galactomannan hydrogel by octadecyl methacrylate as a crosslinking agent has not been reported. The action of octadecyl methacrylate graft on galactomannan with double bond and crosslinking the galactomannan chains by hydrophobic aggregation. This modified galactomannan forms a self-healing hydrogel in aqueous solution. The goal of this work was to prepare the high mechanical strength galactomannan hydrogel with self-healing ability. The structure and characteristics of this modified galactomannan and the influence of hydrophobic aggregation cross-linking in self-healing and mechanical properties were studied.

\section{EXPERIMENTAL}

\section{Materials}

Acrylamide, galactomannan, stearyl methacrylate, ammonium persulfate (APS), sodiumdodecylsulfate (SDS), N,N',N,N'-tetramethylethylenediamine (TEMED), and $\mathrm{NaCl}$ were obtained from Lanyi Chemicals (Beijing, China) and used as received.

\section{Synthesis of Galactomannan-acrylamide-octadecyl Methacrylate Hydrogel}

$\mathrm{NaCl}(0.2925 \mathrm{~g})$ and sodium dodecylsulfate $(0.7 \mathrm{~g})$ were mixed in $20 \mathrm{~mL}$ of galactomannan $(1 \%, \mathrm{w} / \mathrm{v})$ aqueous solution at $60{ }^{\circ} \mathrm{C}$ for $1 \mathrm{~h}$ to obtain a transparent solution. Next, acrylamide (0.9137 g), octadecyl methacrylate $(0.0731 \mathrm{~g})$, TEMED (25 $\mu \mathrm{L})$, and ammonium persulfate $(0.8 \mathrm{~g})$ were added to the solution. The solution was stirred and 
mixed evenly and then poured into the mold. The gel was reacted at room temperature for $24 \mathrm{~h}$ to obtain the hydrogel.

\section{Self-repair Experiment}

The hydrogel self-repair experiment adopts the following methods. When the hydrogel was molded in the mold, a sharp blade was used to cut the gel from the middle. Then, one section was treated with dye (rhodamine B), and the two sections were placed together for $1 \mathrm{~min}, 3 \mathrm{~min}, 5 \mathrm{~min}, 2 \mathrm{~h}, 4 \mathrm{~h}$, and $10 \mathrm{~h}$.

\section{Analytical Methods}

Fourier Transform Infrared (FTIR) spectra were recorded by Fourier transform infrared spectrometer (Tensor27, Nicolet Brooke Company, Karlsruhe, Germany) over the region from 4000 to $400 \mathrm{~cm}^{-1}$. For morphological characterization, the hydrogels were analyzed by scanning electron microscope (SEM) (S-3400N, Hitachi, Tokyo, Japan) with an acceleration voltage of $40 \mathrm{kV}$. A thin layer of the sample was cast on a silica wafer and freeze-dried overnight in a lyophilizer. A layer of gold was sputter-coated over the sample by vacuum spray to form a conductive surface.

Tensile stress-strain measurements were performed on an Instron 3365 Universal Testing Machine (Norwood, MA, USA) with the following parameters: sampling rate, $10.000 \mathrm{pts} / \mathrm{sec}$; beam speed, $100 \mathrm{~mm} / \mathrm{min}$; full-scale load range, $0.1000 \mathrm{kN}$; and temperature, $25^{\circ} \mathrm{C}$. The cylindrical shaped gel samples measured $80 \mathrm{~mm} \times 20 \mathrm{~mm}$. Each data point was measured on six samples, and the average value of five measurements was taken. Statistical analysis of data was performed by one-way analysis of variance, assuming a confidence level of $95 \%(\mathrm{P}<0.05)$ for statistical significance.

The gravimetric method was used to measure the swelling ratios of the gels. After immersion in distilled water for approximately $48 \mathrm{~h}$ at $25^{\circ} \mathrm{C}$ to reach swelling equilibrium, the gel samples were weighed. The average value of three measurements was taken. The equilibrium swelling ratio (SR) was calculated as follows,

$$
\mathrm{SR}=W_{s} / W_{d}
$$

where $W_{s}$ is the weight of the swollen gel and $W_{d}$ is the weight of the gel in its dry state.

\section{RESULTS AND DISCUSSION}

\section{Synthesis of Galactomannan-acrylamide-octadecyl methacrylate Hydrogels}

Galactomannan was used as raw material. Hydrophilic and hydrophobic functional segments were connected by graft polymerization to make a hydrophobically aggregated high-strength galactomannan functional hydrogel. Hydrophobic association of segments is a common method for preparing self-healing hydrogels. The hydrophobic association micelles in the hydrogel network are reversible multi-functional cross-linking points, which act as effective energy dissipation and stress relaxation pathways (Zhu et al. 2018). On the galactomannan macromolecule, the polyacrylamide and poly(stearyl methacrylate grafted sections act as the hydrophilic and hydrophobic segments, respectively. The disordered, freely distributed amphiphility galactomannan macromolecules are guided and regulated by intermolecular hydrophobic interactions, which form a stable crosslinked three-dimensional hydrogel structure (Fig. 1). 


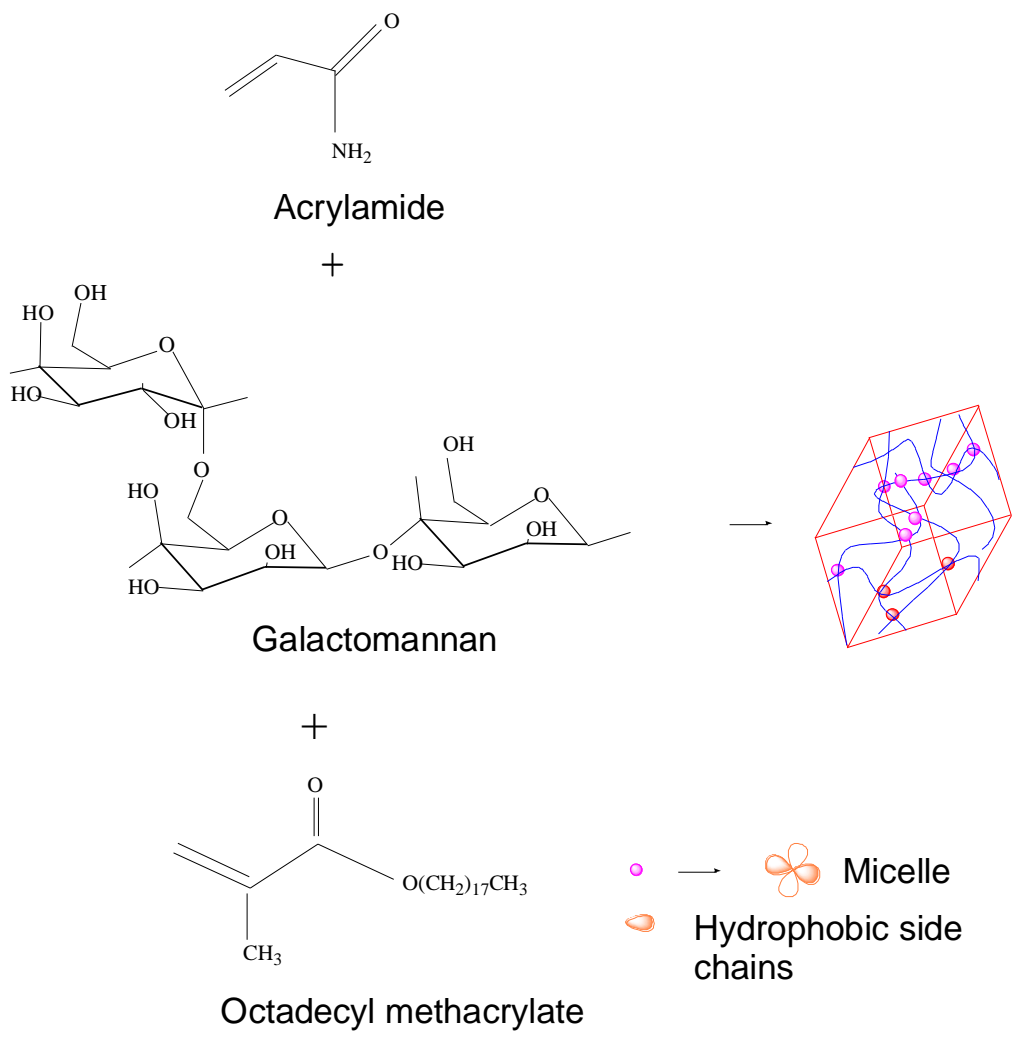

Fig. 1. Synthesis scheme of the galactomannan- acrylamide-octadecyl methacrylate hydrogels

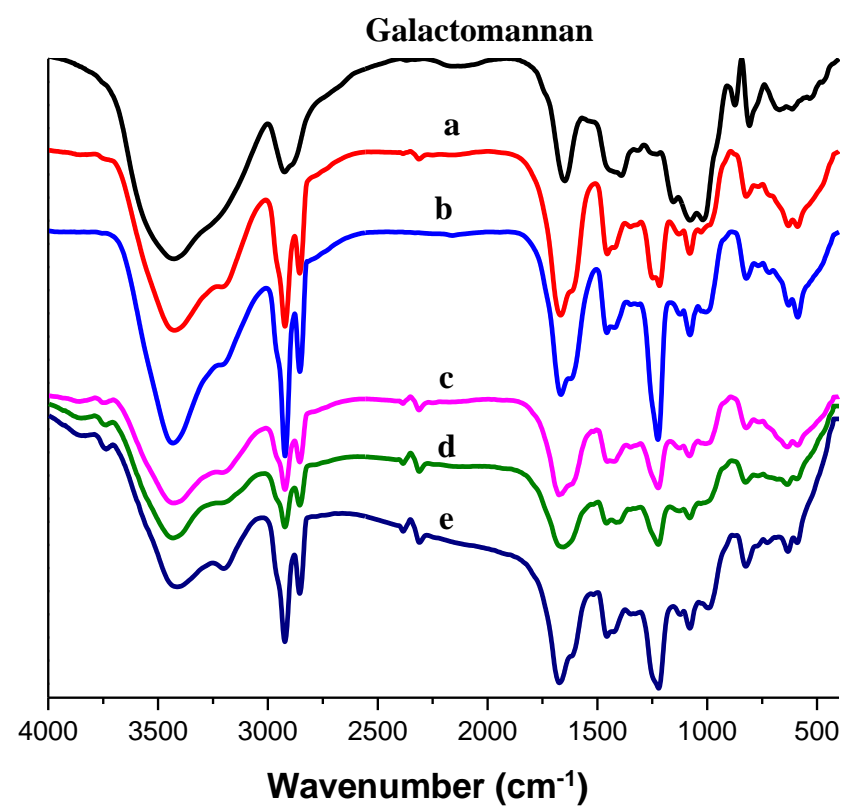

Fig. 2. FTIR spectra of galactomannan-acrylamide-octadecyl methacrylate hydrogels. The mass ratio of octadecyl methacrylate to acrylamide is as follows: (a) $8 \%$, (b) $10 \%$, (c) $12 \%$, (d) $14 \%$, and (e) $16 \%$. 


\section{Characterization of the Galactomannan-acrylamide-octadecyl methacrylate Hydrogels}

FTIR analysis was used to confirm the synthesis of galactomannan and octadecyl methacrylate graft polymer. Figure 2 shows that the galactomannan hydrogels exhibited a broad absorption peak at the wavenumber of 3150 to $3560 \mathrm{~cm}^{-1}$, indicating the hydroxy stretching vibration absorption peak. In Fig. 2 (galactomannan), the curve has a peak at $1654 \mathrm{~cm}^{-1}$ corresponding to the hydroxyl bending (Mahmoud 2016).

However, in the curve of Fig. 2 (a-e), a new sharp and prominent peak located at $1718 \mathrm{~cm}^{-1}$ corresponds to the $\mathrm{C}=\mathrm{O}$ stretching vibration, replacing the absorption band at $1654 \mathrm{~cm}^{-1}$. The stretching vibration peak of C-O-C appeared at $1240 \mathrm{~cm}^{-1}$. The COO-group in the spectrum confirmed the galactomannan successfully formed a crosslinked hydrogel (Thombare et al. 2019).

The elastic stretching vibration peak of a $\mathrm{C}-\mathrm{H}$ bond in the methylene group appeared at $2930 \mathrm{~cm}^{-1}$. The two new peaks at $2930 \mathrm{~cm}^{-1}$ and $1240 \mathrm{~cm}^{-1}$, which can be attributed to the octadecyl methacrylate (Zhu et al. 2018), demonstrate that galactomannan successfully grafted octadecyl methacrylate through graft polymerization initiated by ammonium persulfate.

\section{Mechanical Behavior of the Galactomannan-acrylamide-octadecyl methacrylate Hydrogels}

The ratio of octadecyl methacrylate on acrylamide was changed as $8 \%, 10 \%, 12 \%$, $14 \%$ and $16 \%$. Galactomannan was $1.0 \mathrm{wt} . \%$, and acrylamide was $4.5 \mathrm{wt} . \%$. The amount of octadecyl methacrylate had a remarkable influence on the tensile properties of the hydrogel (Fig. 3a-c). Octadecyl methacrylate provides the hydrophobic aggregate crosslinking point for cross-linked networks. The tensile strength of the hydrogel increased first and then weakened with increasing amounts of octadecyl methacrylate. When octadecyl methacrylate was used at a ratio of $8 \%$, the crosslinking degree is weak, and the hydrogel tensile strength is low (Wu et al. 2008).

The tensile strain exceeded 3000\% (Fig. 3a). The maximum value of tensile stress and the elastic moduli were $0.0488 \mathrm{MPa}$ and $0.00166 \mathrm{MPa}$, respectively, at the $10 \%$ ratio of octadecyl methacrylate. The strain showed the highest value at $8 \%$ octadecyl methacrylate. The increasing hydrogel strength reflects the increase in crosslink density, which reduces the space between the grids of the three-dimensional network. The individual polymers are tightly bonded or joined to each other, making the hydrogel harder and more compact. However, too many cross-linking points decrease the strength because the network structure is too dense (Thombare et al. 2019).

The galactomannan-acrylamide-octadecyl methacrylate hydrogel had good compression properties, and the amount of octadecyl methacrylate had little effect on the compression performance. Figures $3 \mathrm{~d}$ and $3 \mathrm{e}$ show that the compressive strain of the hydrogel changed from $92 \%$ to $97 \%$ when the ratio of octadecyl methacrylate to acrylamide increased from $8 \%$ to $16 \%$. The highest elastic modulus $0.06451 \mathrm{MPa}$ was achieved with an $8 \%$ ratio of octadecyl methacrylate. As the amount of octadecyl methacrylate increased, excessive cross-linking resulted in a harder hydrogel and a more compact threedimensional structure. 
a
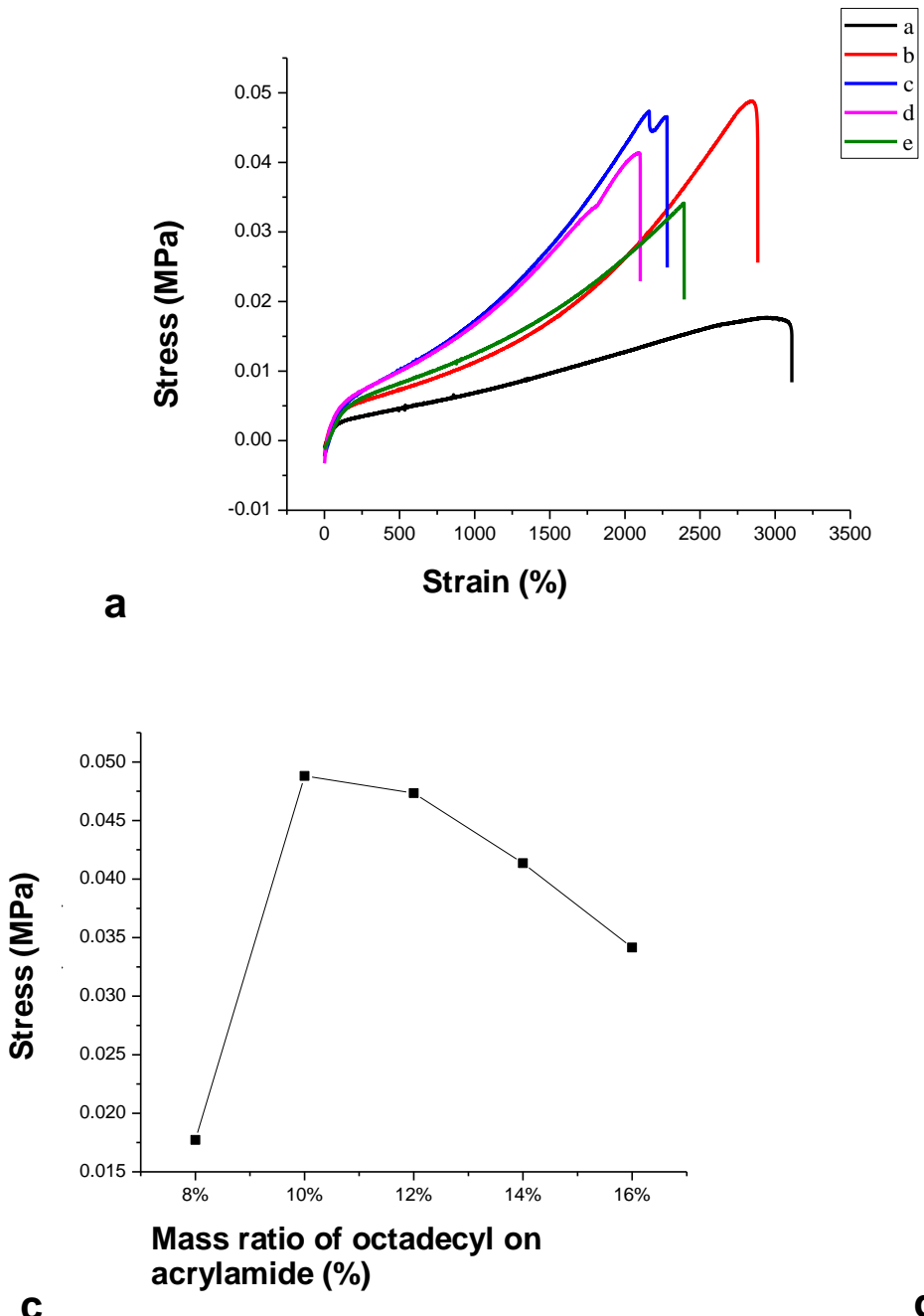

b

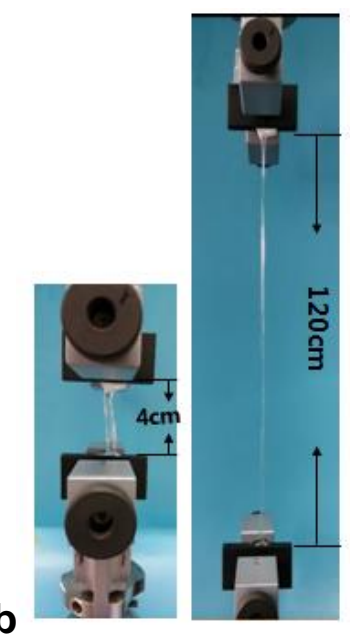

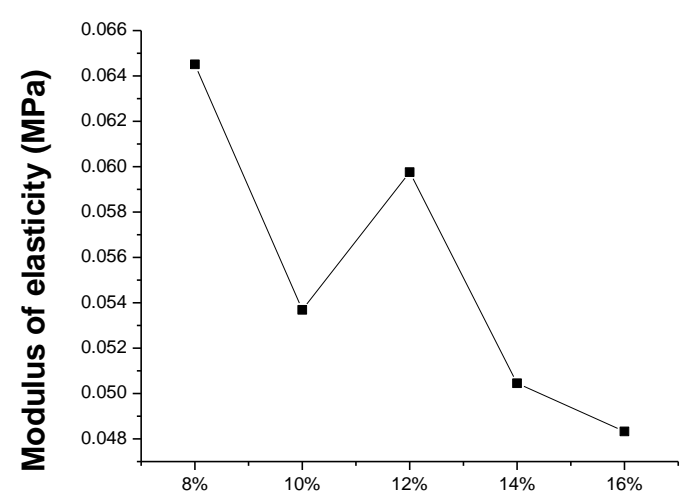

Mass ratio of octadecyl methacrylate on acrylamide (\%)

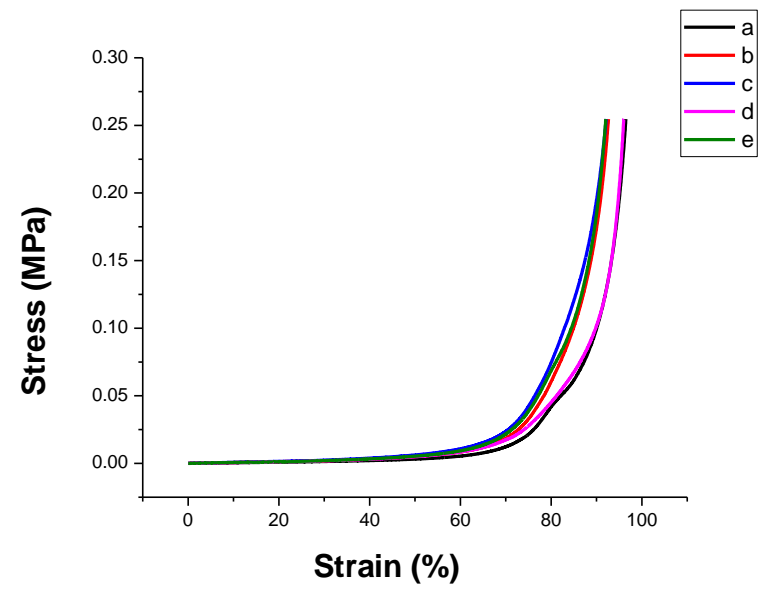

d 


\section{Adsorption Performance of the Galactomannan-acrylamide-octadecyl methacrylate Hydrogels}

Hydrogel is a soft and wet material with three-dimensional networks, which can absorb large amounts of water. Galactomannan forms cross-linking points on the hydrophobic side chain of octadecyl methacrylate and connects polymers to form a threedimensional network with internal pore space. Water absorption is an important property of hydrogel materials. Galactomannan-octadecyl methacrylate hydrogels swell and absorb water in aqueous solution with good hydrophilicity. The water absorption capacity of the galactomannan-octadecyl methacrylate hydrogels was studied in distilled water.
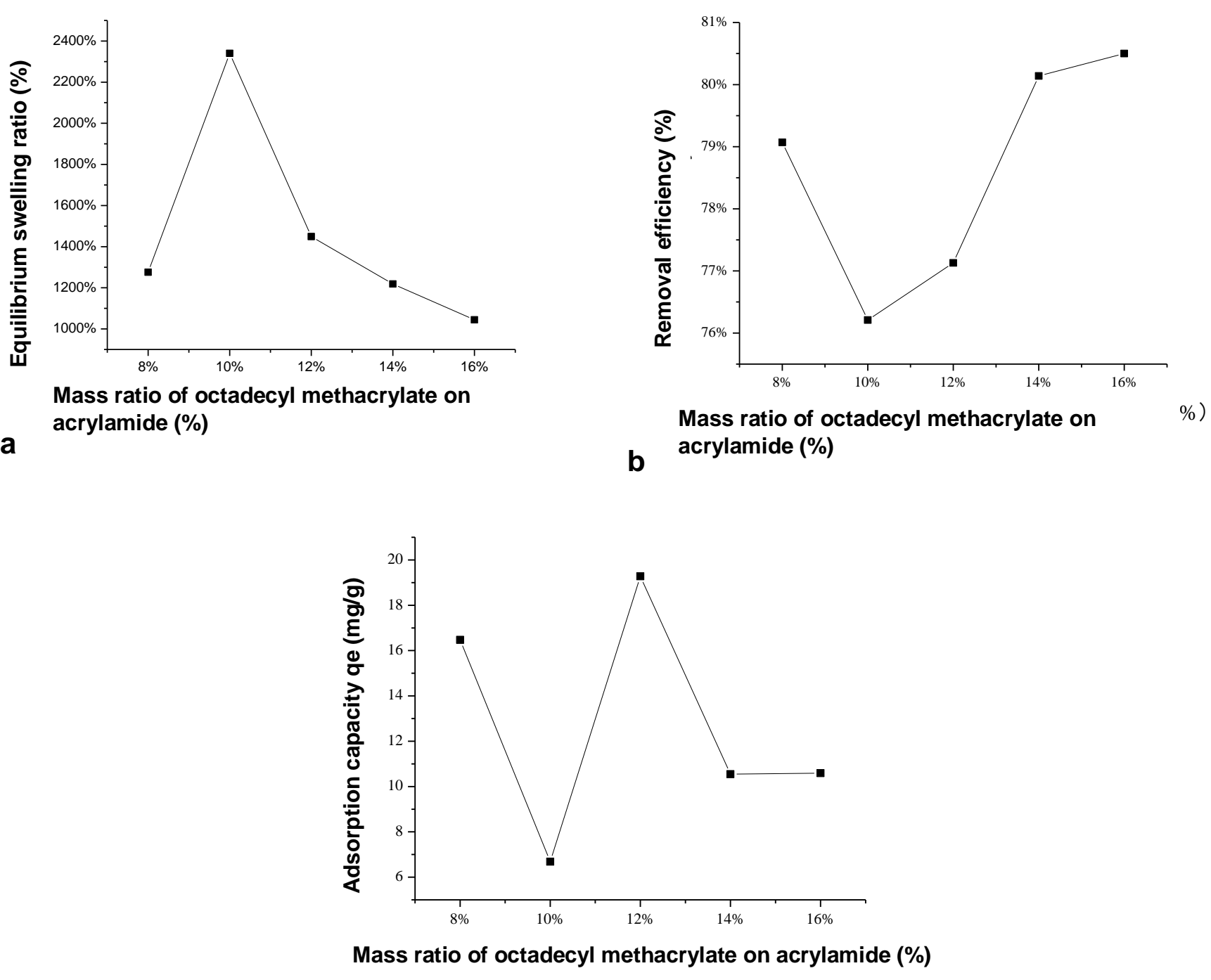

C

Fig. 4. Adsorption performance of the galactomannan-acrylamide-octadecyl methacrylate hydrogels. Effect of the mass ratio of octadecyl methacrylate on (a) the equilibrium swelling ratio and $(b, c)$ the adsorption properties of methylene blue.

The amount of octadecyl methacrylate reflects the relationship between the crosslink density and the water absorption capacity of the hydrogel. As shown in Fig. 4a, the water absorption rate increased first and then decreased with the increasing mass ratio of 
octadecyl methacrylate on acrylamide from $8 \%$ to $16 \%$. The highest water absorption reached $2340 \%$ at the $10 \%$ octadecyl methacrylate ratio. When the amount of octadecyl methacrylate was increased, the cross-linking point and crosslink density increased. Correspondingly, hydrogel swelling behavior is affected by crosslink density. The moisture initially fills the large pores within the gel and then gradually fills the micropores so that the hydrogel absorbs a large amount of water. The individual polymer segments are tightly bonded or joined to each other, and the pore space in the three-dimensional structure is greatly reduced, which limits the entry of water into the gel and the gel expansion ability. Thus, when the amount of octadecyl methacrylate increased from $10 \%$ to $16 \%$, the hydrogel water absorption rate decreased. When methylene blue was used as the target adsorbent of hydrogels, the removal rate first decreased and then increased with the increased octadecyl methacrylate content (Fig.4b,c). When the ratio of octadecyl methacrylate to acrylamide was $16 \%$, the removal rate reached a maximum of $80.5 \%$. When the ratio was $12 \%$, the adsorption capacity reached a maximum of $19.3 \mathrm{mg} / \mathrm{g}$. The swelling property of hydrogel is one of the factors related to its adsorption properties. The swelling rate of hydrogel is large, and its internal gap is large, which is more conducive to the adsorption of adsorbed substances inside the hydrogel. In the initial stage, the swelling rate of hydrogels increased linearly, because the molecular chains of dry hydrogels were tightly packed before swelling. After immersion in the solution, the water molecules in the solution interact with the polar groups of the amide groups and hydroxyl groups on the gel to form intermolecular hydrogen bonds, thus reducing the interaction force between the macromolecular network segments and expanding the network. Then the network molecular chain extends to the three-dimensional space of the gel, the hydrogen bonds between $-\mathrm{NH}_{2}$ and $-\mathrm{OH}$ break down gradually, which makes the swelling accelerate automatically. With the further acceleration of the swelling process, the space of the water molecules in the gel gradually decreases, and the swelling rate tends to slow until the swelling equilibrium is reached.

\section{Self-healing Properties of the Galactomannan-acrylamide-octadecyl methacrylate Hydrogels}

The galactomannan-acrylamide-octadecyl methacrylate hydrogel exhibited good self-healing properties. As shown in Fig. 5a, after the gel was cut in half, the two sections self-healed in a few minutes at room temperature without complicated treatment. The reversible hydrophobic aggregation of the hydrogel contributes to the good self-healing property (Zhu et al. 2018). Once the two sections of the hydrogel cut surface are contacted, the molecular chains at the cut surface begin to mutually diffuse and the hydrophobic parts can form new cross-linking points, the three-dimensional network of the hydrogel will be reconstituted (Duan et al. 2014 a,b; Taylor and In Het Panhuis 2016). After the hydrogel was cut and dyed, the hydrogels were connected for $10 \mathrm{~h}$ and $16 \mathrm{~h}$, respectively (Fig. $5 \mathrm{~b}$ ). The red dye moved toward the unstained half of the gel. The hydrogel had good molecular diffusion and migration behavior, and the hydrogel cross section achieved good fusion. When the hydrogel was exposed to air for $10 \mathrm{~h}$ at room temperature, the hydrogel cut surfaces disappeared, and the hydrogel self-healed autonomously. The tensile fracture strength of the self-healed hydrogel increased gradually with prolonged contact time (Fig. $5 \mathrm{c})$. The molecular diffusion of the cut surface was the main reason for the improvement of hydrogel self- repairing strength. 
a
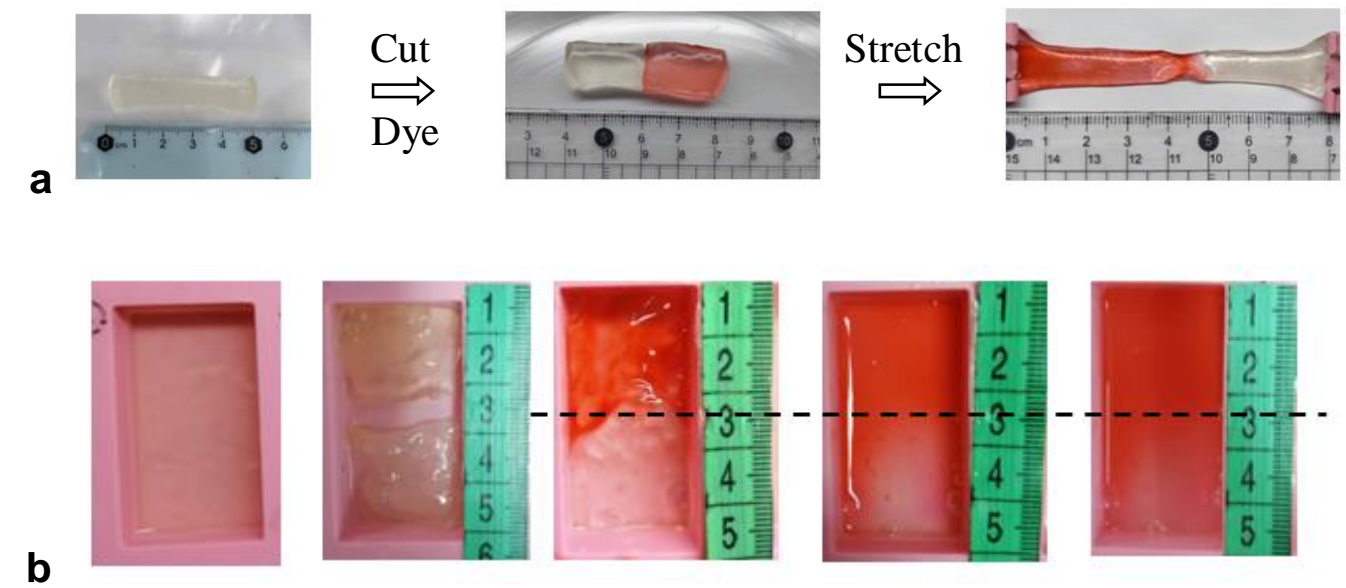

Original

Cut

Dye

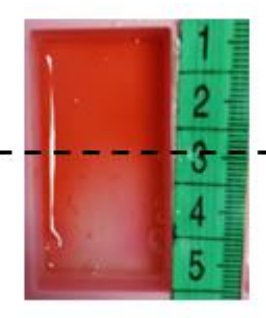

$10 \mathrm{~h}$

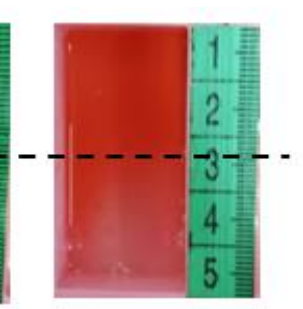

$16 \mathrm{~h}$

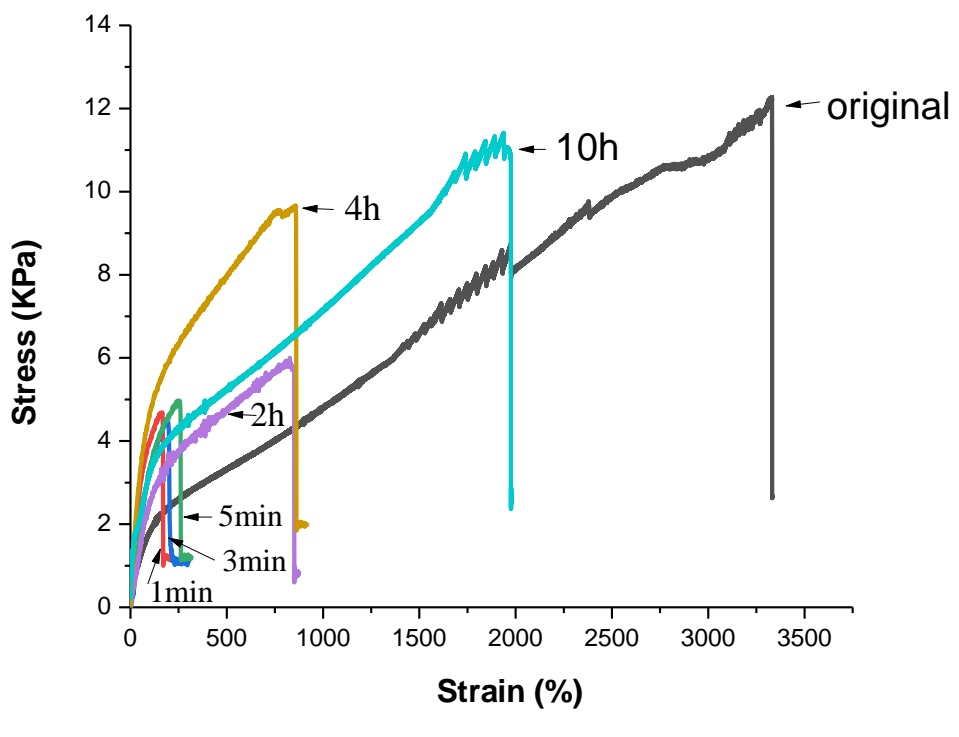

C

Fig. 5. Self-healing property of the galactomannan-acrylamide-octadecyl methacrylate hydrogels

\section{Hydrogel Morphology}

The prepared hydrogel was vacuum freeze-dried for $48 \mathrm{~h}$, and the dried hydrogel was subjected to gold spraying treatment after being cut. The treated galactomannan hydrogel was observed by SEM. The single polymer of the hydrogel was connected by hydrophobic aggregation cross-linking points forming a three-dimensional network structure with internal pore space. Figure 6 shows that the hydrogel had a porous structure, a spongy surface, and a lot of internal space. This hydrogel morphology allows direct penetration of water and promotes the diffusion of aqueous fluids. This morphology contributes to the good adsorption and swelling properties of the hydrogel. Specific surface area is important for the application of materials (Zhang et al. 2017, 2018). The porous structure of hydrogels has a large specific surface area, which may be one of the reasons for the high water absorption of galactomannan- acrylamide- octadecyl methacrylate hydrogels. 


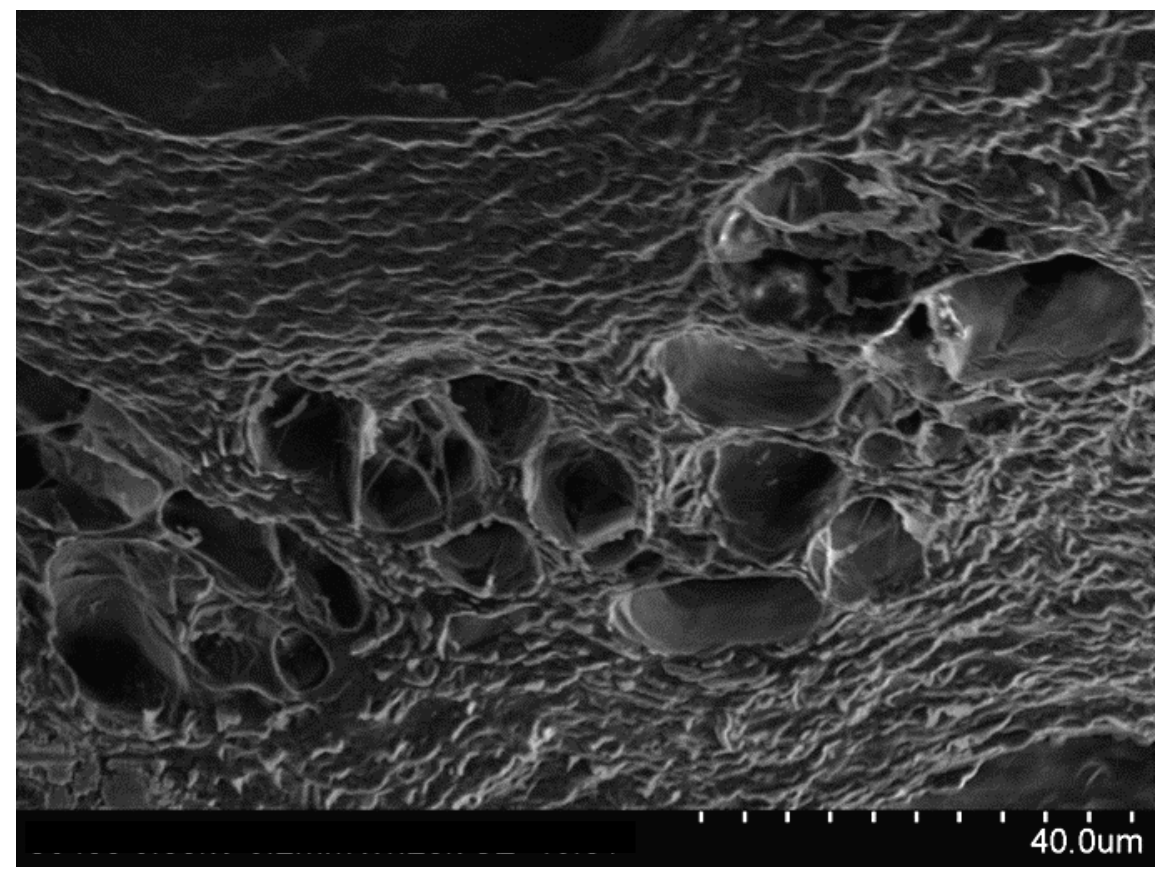

Fig. 6. Scanning electron micrograph of galactomannan- acrylamide- octadecyl methacrylate hydrogel

\section{CONCLUSIONS}

1. Galactomannan is a suitable replacement for conventional petroleum resources in the preparation of biopolymer-based plastics. This study shows a new application of galactomannan.

2. A progressive self-repairing galactomannan hydrogel was prepared by solution polymerization. The hydrogel fracture surface self-healed in a few seconds at room temperature. The grafted hydrophobic chain poly(octadecyl methacrylate) aggregation becomes the cross-linking point of the three-dimensional network of galactomannan hydrogel. The molecular chains of the cut surface of the hydrogel can diffuse and reform the hydrophobic aggregation cross-linking points, and this is the main reason for the self-healing. The healing efficiency of galactomannan- acrylamide-octadecyl methacrylate hydrogel is affected by healing time, and the fracture strain can reach $92.7 \%$ of the original sample in $10 \mathrm{~h}$.

3. The hydrogel exhibited a maximum water absorption of $2340 \%$. The mechanical strength of this hydrogel reached $0.0488 \mathrm{MPa}$, and it maintained its integrity under compression up to the compression deformation of $98 \%$. The maximum compressive elastic modulus was $0.0645 \mathrm{MPa}$. The tensile strain exceeded $3000 \%$.

\section{ACKNOWLEDGMENTS}

This research was financially supported by the National Natural Science Foundation of China (31600464) and the China Ministry of Science and Technology (2016YFD0600803). 


\section{REFERENCES CITED}

Ali, M., and Husain, Q. (2018). "Guar gum blended alginate/agarose hydrogel as a promising support for the entrapment of peroxidase: Stability and reusability studies for the treatment of textile effluent," International Journal of Biological Macromolecules 116 (2018), 463-471. DOI: 10.1016/j.ijbiomac.2018.05.037 01418130

Bahamdan, A., and Daly, W. H. (2007). "Hydrophobic guar gum derivatives prepared by controlled grafting processes - Part II: Rheological and degradation properties toward fracturing fluids applications," Polymers for Advanced Technologies 18(8), 660-672. DOI:10.1002/pat.875

Bai, T., Liu, S., Sun, F., Sinclair, A., Zhang, L., Shao, Q., and Jiang, S. (2014). "Zwitterionic fusion in hydrogels and spontaneous and time-independent self-healing under physiological conditions," Biomaterials 35(13), 3926-3933. DOI: 10.1016/j.biomaterials.2014.01.077

Bak, J. H., and Yoo, B. (2018). "Intrinsic viscosity of binary gum mixtures with xanthan gum and guar gum: Effect of $\mathrm{NaCl}$, sucrose, and $\mathrm{pH}$," International Journal of Biological Macromolecules 111, 77-81. DOI: 10.1016/j.ijbiomac.2017.12.144

Barcan, G. A., Zhang, X., and Waymouth, R. M. (2015). "Structurally dynamic hydrogels derived from 1,2-dithiolanes," Journal of the American Chemical Society 137(17), 5650-5653. DOI:10.1021/jacs.5b02161

Bizien,T., Ameline, J. C., Yager, K. G., Marchi, V., and Artzner, F. (2015). "Selforganization of quantum rods induced by lipid membrane corrugations," Langmuir 31(44), 12148-12154. DOI: 10.1021/acs.langmuir.5b03335

Chaurasia, M., Chourasia, M. K., Jain, N. K., Jain, A., Soni, V., Gupta, Y., and Jain, S. K. (2006). "Cross-linked guar gum microspheres: A viable approach for improved delivery of anticancer drugs for the treatment of colorectal cancer," AAPS PharmSciTech 7(3), 143-151. DOI: 10.1208/pt070374

Chaires-Martinez, L., Salazar-Montoya, J. A., and Ramos-Ramirez, E. G. (2008). "Physicochemical and functional characterization of the galactomannan obtained from mesquite seeds (Prosopis pallida)," European Food Research and Technology 227(6), 1669-1676. DOI: 10.1007/s00217-008-0892-0

Chirila, T. V., Lee, H. H., Oddon, M., Nieuwenhuizen, M. M. L., Blakey, I., and Timothy, M. (2014). "Hydrogen-bonded supramolecular polymers as self-healing hydrogels: Effect of a bulky adamantyl substituent in the ureido-pyrimidinone monomer," Journal of Applied Polymer Science 134(4), 1-12. DOI: 10.1002/app.39932

Cui, J., Wang, M., Zheng, Y., Muñiz, G. M. R., and Campo A. (2013). "Light-triggered cross-linking of alginates with caged $\mathrm{Ca}^{2+}$," Biomacromolecules 14(5), 1251-1256. DOI: $10.1021 / \mathrm{bm} 400022 \mathrm{~h}$

Cunha, P. L. R., Castro, R. R., Rocha, F. A. C., Paula, R. C. M. D., and Feitosa, J. P. A. (2005). "Low viscosity hydrogel of guar gum: Preparation and physicochemical characterization," International Journal of Biological Macromolecules 37(1-2), 99104. DOI: 10.1016/j.ijbiomac.2005.09.001 
Deng, G., Li, F., Yu, H., Liu, F., Liu, C., Sun, W., Jiang, H., and Chen, Y. (2012).

"Dynamic hydrogels with an environmental adaptive self-healing ability and dual responsive sol-gel transitions," ACS Macro Letters 1(2), 275-279. DOI:

$10.1021 / \mathrm{mz} 200195 \mathrm{n}$

Duan, J. F., and Jiang, J. X. (2017). "Structure and properties of hydrophobic aggregation hydrogel with chemical sensitive switch," International Journal of Polymer Science 2017, 1-5. DOI:10.1155/2017/9123248

Duan, J. F., Zhang, X. J., Jiang, J. X., Han, C. R., Yang, J., Liu, L. J., Lan, H. Y., and Huang, D. Z. (2014a). "The synthesis of a novel cellulose physical gel," Journal of Nanomaterials 2014, 1-7. DOI: 10.1155/2014/312696

Duan, J. F., Jiang, J. X., Han, C. R., Yang, J., Liu, L. J., and Li, J. Z. (2014b). "The study of intermolecular inclusion in cellulose physical gels," BioResources 9(3), 40064013. DOI: 10.15376/biores.9.3.4006-4013

Fu, L., Wu, C., Zhou, Y., Zuo, J., and Song, G. (2019). "Effects of residual ozone on the performance of microorganisms treating petrochemical wastewater," Environmental Science and Pollution Research, 26(26), 27505-27515. DOI: 10.1007/s11356-01905956-8

Li, Y. P., Liu, Y .C., Tang, H. B., and Dong, S. Q. (2018). “Oxidized cross-linked guar gum with hydrophobic groups: Structure, properties and removal of Reactive BlueXBR in simulated water," Arabian Journal for Science and Engineering 43(7), 36213629. DOI: 10.1007/s13369-018-3146-X

Mukherjee, S., Mukhopadhyay, S., Bin Zafri, M. Z., Zhan, X. M., Hashim, M. A., and Sen Gupta, B. (2018). "Application of guar gum for the removal of dissolved lead from wastewater," Industrial Crops and Products 111 (2018), 261-269.

DOI:10.1016/j.indcrop.2017.10.022

Paixao, M. V. G., and Balaban, R. D. (2018). "Application of guar gum in brine clarification and oily watertreatment," International Journal of Biological Macromolecules 108, 119-126. DOI: 10.1016/j.ijbiomac.2017.11.166

Saurabh, C. K., Gupta, S., and Variyar, P. S. (2018). "Development of guar gum based active packaging films using grape pomace," Journal of Food Science and Technology 55(6), 1982-1992. DOI: 10.1007/s13197-018-3112-3

Shi, X. N., Wang, W. B., and Wang, A. Q. (2011). "Synthesis and enhanced swelling properties of a guar gum-based superabsorbent composite by the simultaneous introduction of styrene and attapulgite," Journal of Polymer Research 18(6), 17051713. DOI: $10.1007 / \mathrm{s} 10965-011-9576-8$

Mahmoud, R. I. (2016). "Investigation of partially methylolated polyacrylamide guar gum by FTIR and thermal properties," British Journal of Applied Science \&Technology 2016, 1-11. DOI:10.9734/BJAST/2016/28761

Tang, H. B., Sun, M., Li, Y. P., and Dong, S. Q. (2013). "Preparation and properties of partially hydrolyzed cross-linked guar gum," Polymer Bulletin 70(12), 3331-3346. DOI: $10.1007 / \mathrm{s} 00289-013-1025-\mathrm{x}$

Taylor, D. L., and In Het Panhuis, M. (2016). "Self-healing hydrogels," Advanced Materials 28(41), 9060-9093. DOI: 10.1002/adma.201601613

Thombare, N., Mishra, S., Siddiqui, M. Z., Jha, U., Singh, D., and Mahajan, G. R. (2018). "Design and development of guar gum based novel, superabsorbent and moisture retaining hydrogels for agricultural applications," Carbohydrate Polymers 185,169178. DOI: 10.1016/j.carbpol.2018.01.018 
Tuncaboylu, D. C., Sahin, M., Argun, A., Oppermann, W., and Okay, O. (2012). "Dynamics and large strain behavior of self-healing hydrogels with and without surfactants," Macromolecules 45(4), 1991-2000. DOI: 10.1016/j.carbpol.2018.01.018

Wang, G. W., Wei, X. J., Tanaka, T., and Kataura, H. (2018). "Facile synthesis of guar gum gel for the separation of metallic and semiconducting single-wall carbon nanotubes," Carbon 129, 745-749. DOI: 10.1016/j.carbon.2017.12.064

Wang, Y., Xiong, Y., Wang, J., and Zhang, X. (2017). "Ultrasonic-assisted fabrication of montmorillonite-lignin hybrid hydrogel: Highly efficient swelling behaviors and super-sorbent for dye removal from wastewater," Colloids and Surfaces A:

Physicochemical and Engineering Aspects 520, 903-913. DOI:

10.1016/j.colsurfa.2017.02.050

Wang, Y., Wang, Y., Yu, L., Wang, J., Du, B., and Zhang, X. (2019). "Enhanced catalytic activity of templated-double perovskite with 3D network structure for salicylic acid degradation under microwave irradiation: Insight into the catalytic mechanism," Chemical Engineering Journal 368, 115-128. DOI:

10.1016/j.cej.2019.02.174

Wei, Z., He, J., Liang, T., Oh, H., Athas, J., Tong, Z., Wang, C., and Nie, Z. (2013). "Autonomous self-healing of poly(acrylic acid) hydrogels induced by the migration of ferric ions," Polymer Chemistry 4(15), 4061-4065. DOI: 10.1039/c3py00692a

Wei, Z., Yang, J. H., Liu, Z. Q., Xu, F., Zhou, J. X., Zrınyi, M., Osada, Y., and Chen, Y. M. (2015). "Novel biocompatible polysaccharide-based self-healing hydrogel," Advanced Functional Materials 25(9), 1352-1359. DOI: 10.1002/adfm.201401502

Wu, L., and Liu, M. (2008). "Preparation and properties of chitosan-coated NPK compound fertilizer with controlled-release and water-retention," Carbohydrate Polymers 72(2), 240-247. DOI: 10.1016/j.carbpol.2007.08.020

Yang, Y., Ding, Q., Wen, D., Yang, M., Wang, Y., Liu, N., and Zhang, X. (2018). "Bromate removal from aqueous solution with novel flower-like Mg-Al-layered double hydroxides," Environmental Science and Pollution Research, 25(27), 2750327513. DOI: $10.1007 / \mathrm{s} 11356-018-2781-9$

Zhang, X., Li, H., Hou, F., Yang, Y., Dong, H., Liu, N., Wang, Y., and Cui, L. (2017). "Synthesis of highly efficient $\mathrm{Mn}_{2} \mathrm{O}_{3}$ catalysts for $\mathrm{CO}$ oxidation derived from MnMIL-100," Applied Surface Science, 411, 27-33. DOI: 10.1016/j.apsusc.2017.03.179

Zhang, X., Wang, Y., Hou, F., Li, H., Yang, Y., Zhang, X., Yang, Y., and Wang, Y. (2017). "Effects of Ag loading on structural and photocatalytic properties of flowerlike ZnO microspheres," Applied Surface Science, 391, 476-483. DOI: 10.1016/j.apsusc.2016.06.109

Zhang, X., Zhang, X., Song, L., Hou, F., Yang, Y., Wang, Y., and Liu, N. (2018). "Enhanced catalytic performance for $\mathrm{CO}$ oxidation and preferential $\mathrm{CO}$ oxidation over $\mathrm{CuO} / \mathrm{CeO}_{2}$ catalysts synthesized from metal organic framework: Effects of preparation methods," International Journal of Hydrogen Energy, 43(39), 1827918288. DOI: 10.1016/j.ijhydene.2018.08.060

Zhang, X., Yang, Y., Lv, X., Wang, Y., Liu, N., Chen, D., and Cui, L. (2019). "Adsorption/desorption kinetics and breakthrough of gaseous toluene for modified microporous-mesoporous UiO-66 metal organic framework," Journal of Hazardous Materials, 366, 140-150. DOI: 10.1016/j.jhazmat.2018.11.099 
Zhang, X., Yang, Y., Huang, W., Yang, Y., Wang, Y., He, C., Liu, N., Wu, M., and Tang, M. (2018). "g-C3N4/UiO-66 nanohybrids with enhanced photocatalytic activities for the oxidation of dye under visible light irradiation," Materials Research Bulletin 99, 349-358.

Zhu, J., Guo, P., Chen, D., Xu, K., Wang, P., and Gua, S. (2018). "Fast and excellent healing of hydroxypropyl guar gum/poly(N,Ndimethyl acrylamide) hydrogels," Journal of Polymer Science Part B: Polymer Physics 56(3), 239-247. DOI: 10.1002/polb.24514

Article submitted: July 25, 2019; Peer review completed: October 19, 2019; Revise version received: October 22, 2019; Accepted: October 23, 2019; Published: October 28, 2019.

DOI: $10.15376 /$ biores.14.4.9853-9866 